\title{
Micronized purified flavonoid fraction in hemorrhoid disease: a systematic review and meta-analysis
}

\section{Parvez Sheikh \\ Varut Lohsiriwat \\ Yury Shelygin}

\section{Video Abstract}

Keywords: hemorrhoids, hemorrhoidal disease, hemorrhoidectomy, venoactive drugs, micronized purified flavonoid fraction, MPFF, systematic review, randomized clinical trial, pain, bleeding, pruritus, discharge, leakage, tenesmus, edema, meta-analysis, venous blood, prolapse, phlebotonics, Daflon

Posted Date: July 27th, 2020

DOI: https://doi.org/10.21203/rs.3.rs-49672/v1

License: (9) This work is licensed under a Creative Commons Attribution 4.0 International License.

Read Full License 


\section{Abstract}

A new study investigated the efficacy of micronized purified flavonoid fraction, or MPFF, in the treatment of patients with hemorrhoidal disease. Hemorrhoidal disease affects an estimated $4.4 \%$ of the global population. The disease occurs when hemorrhoids become inflamed and swollen with venous blood. While severe cases are typically treated with surgery, banding, or other outpatient procedures, some cases can be treated conservatively, through diet, lifestyle modification, topical treatments, or agents known as veno-active drugs. Veno-active drugs are designed to reduce inflammation and reduce pressure in veins, improving their overall tone and translating to symptom relief in patients. One evidence based veno-active treatment for hemorrhoids is MPFF. MPFF has been shown to be effective against inflammation associated with venous conditions such as varicose veins, and in patients with hemorrhoidal disease, previous studies have shown that MPFF reduces pain, bleeding, anal discharge, and prolapse, and is effective in preventing relapse and reducing the duration and severity of acute attacks. This latest study aimed to confirm these findings across a broader spectrum of signs and symptoms following MPFF treatment in patients with hemorrhoidal disease. A systematic review of the literature was performed to identify randomized clinical trials in which MPFF treatment was compared with placebo or no treatment for acute hemorrhoidal disease or for relief of symptoms after patients had undergone medical management or a surgical procedure to remove hemorrhoids. A total of 497 records were identified, of which 11 studies reported in 13 articles were included in the review and 4 were included in the metaanalysis. These four studies examined one of two different situations of use for MPFF: acute hemorrhoidal disease or a medical or surgical procedure for hemorrhoidal disease. All studies were single- or double-blind randomized controlled trials comparing MPFF with placebo or no treatment. Participants included patients of both sexes, including pregnant or post-partum women, and patients undergoing a procedure for hemorrhoidal disease. The main efficacy endpoints were bleeding, pain, and itch. Other outcomes included analgesic consumption, discharge or leakage, overall improvement according to patient or investigator, anal discomfort, edema, tenesmus, recurrence of hemorrhoidal crisis, and length of hospital stay following a procedure. Quantitative analysis of various studies indicated that MPFF provided significant benefits across several signs and symptoms of acute hemorrhoidal disease. For bleeding, two studies were included in the pooled analysis, and three were included in the sensitivity analysis. Both analyses indicated that MPFF has a statistically significant effect on bleeding. For anal discharge and leakage, two studies were included in the meta-analysis. The meta-analysis indicated that MPFF has a statistically significant effect on discharge and leakage. Pooled meta-analyses of three different studies indicated overall improvement as reported by patients and also by investigators. The meta-analysis also showed that treatment with MPFF tended to reduce pain. Even though the results could not be pooled for some parameters due to differences in reporting units, qualitative data for all parameters were presented that provided new information in a broad perspective. Reduction in the need for concomitant analgesic use, shorter hospital stays after hemorrhoidectomy, and prevention of relapse have been identified as indirect benefits of treatment with MPFF. Taken together, the results indicated that MPFF treatment can improve the most important signs and symptoms of hemorrhoidal disease, including bleeding, pain, pruritus, tenesmus, and anal discharge/leakage. 